\title{
Quantum mechanical and astrophysical studies of methanol
}

\author{
David Flower ${ }^{1, a}$, D. Rabli ${ }^{1}$, and G. Pineau des Forêts ${ }^{2}$ \\ 1 Physics Department, The University, Durham DH1 3LE, UK \\ 2 IAS (UMR 8617), Université de Paris-Sud, F-91405 Orsay, France
}

\begin{abstract}
Interstellar methanol has been observed extensively at radio frequencies from the ground, and, very recently, it has been observed at higher frequencies, by means of the Herschel satellite. Being a complex molecule, methanol has a rich spectrum exhibiting rotational and internal torsional motions. However, by the same token, the determination of the cross sections and rate coefficients for the excitation of methanol by the principal perturbers, helium and molecular hydrogen, is a far from trivial task. We have recently extended and considerably improved previous calculations of these data. In the case of molecular hydrogen, results are now available for the excitation of both A- and E-type methanol, not only by para- but also by ortho- $\mathrm{H}_{2}$. These data have been used to model the HIFI observations of the outflow source L1157 B1. The methanol emission is computed self-consistently, in parallel with the dynamics and the chemistry, allowing for the optical depths in the emission lines by means of the LVG approximation. The results of these calculations are summarized.
\end{abstract}

\section{Introduction}

Interstellar methanol $\left(\mathrm{CH}_{3} \mathrm{OH}\right)$ has been observed extensively from the ground, at radio wavelengths. It exhibits population inversion (maser action) and anti-inversion (absorption of the microwave background radiation). Very recently, methanol has been observed, in the molecular outflow L1157 B1, at higher frequencies, by means of the HIFI instrument, aboard the Herschel satellite [1]. The observed lines are believed to be excited by a shock wave.

\section{Structure of methanol}

To a first approximation, methanol may be considered to be a symmetric top. This approximation would be exact if the axis of the $\mathrm{OH}$ radical in the $\mathrm{CH}_{3} \mathrm{OH}$ structure was aligned exactly with the three-fold symmetry axis of the $\mathrm{CH}_{3}$ group. In reality, the $\mathrm{O}-\mathrm{H}$ axis is slightly inclined, relative to this symmetry axis, making methanol an asymmetric top.

However, there is a further complication: the $\mathrm{CH}_{3}$ group can rotate internally, relative to the $\mathrm{OH}$ radical, around the $\mathrm{CH}_{3}$ symmetry axis; this motion is termed "torsional". Torsional motion is analogous to the vibrational motion of molecules, but the energy required to excite torsional motion is of the order of $100 \mathrm{~cm}^{-1}$, as compared with the value of $1000 \mathrm{~cm}^{-1}$ that is more typical of linear vibrations. Consequently, the rotational levels belonging to different torsional states overlap at energies that are readily attainable in shock-heated gas.

Methanol exists in two nuclear-spin states, depending on the net spin of the three protons of the $\mathrm{CH}_{3}$ group. In A-type methanol (analogous to ortho- $\mathrm{NH}_{3}$ ), the proton spins are parallel, and $I=\frac{3}{2}$. In E-type methanol (analogous to para-NH3), one of the proton spins is anti-parallel, and $I=\frac{1}{2}$.

\footnotetext{
a e-mail: david.flower@durham.ac.uk
} 
Furthermore, E-type methanol has two exactly degenerate forms, $\mathrm{E}_{1}$ and $\mathrm{E}_{2}$. Consequently, the relative statistical abundance ratio of A-type to E-type is not the ratio of the spin degeneracies (2), but 1 .

\section{Recent calculations of rate coefficients}

We summarize briefly here the data that are now available online ${ }^{1}[2-4]$.

- $\mathrm{CH}_{3} \mathrm{OH}-\mathrm{He}$ : rotational transitions between 256 levels of A-type and 256 levels of E-type in their ground torsional states $(T \leq 200 \mathrm{~K})$; rotational transitions between 150 levels of A-type and 150 levels of E-type in their ground and first two excited torsional states $(T \leq 400 \mathrm{~K})$.

- $\mathrm{CH}_{3} \mathrm{OH}-\mathrm{H}_{2}$ : rotational transitions between 256 levels of A-type and 256 levels of E-type in their ground torsional states, induced by para- $\mathrm{H}_{2}(J=0)(T \leq 200 \mathrm{~K})$; rotational transitions between 100 levels of A-type and 100 levels of E-type in their ground torsional states, induced by ortho- $\mathrm{H}_{2}$ $(J=1)(T \leq 200 \mathrm{~K})$.

\section{Shock-wave model}

Methanol has been incorporated into our MHD shock wave code, and its predictions were compared with the Herschel HIFI spectrum of L1157 B1 [5]. The equations for the escape probabilities and level populations were integrated in parallel with the dynamical and chemical rate equations. The rate of radiative cooling was calculated self-consistently. Ortho- and para- $\mathrm{H}_{2} \mathrm{O}$ and $\mathrm{NH}_{3}$, and also $\mathrm{CO}$ and $\mathrm{SiO}$, were treated analogously.

- We find that the transitions $\mathrm{A}^{+} 7_{0} \rightarrow 6_{1}(44.07 \mathrm{GHz})$ and $\mathrm{E} 4_{-1} \rightarrow 3_{0}(36.17 \mathrm{GHz})$, which have optical depths (at the line centres) attaining -4.1 and -3.3 , respectively, are the most intense lines of the respective methanol types. These transitions have been observed recently in a Class I methanol maser source, in which the emission appears to be related to an outflow along the line of sight and an associated shock wave [6].

- Several transitions appear in absorption against the cosmic background radiation, notably E $2_{0} \rightarrow$ $3_{-1}(12.18 \mathrm{GHz})$, observed by Walmsley et al. (1988) [7] to be anti-inverted towards dark clouds. The integrated intensity of this line is $T \mathrm{~d} V=-26.2 \mathrm{~K} \mathrm{~km} \mathrm{~s}^{-1}$. In addition, the $\mathrm{A}^{+} 4_{1} \rightarrow 5_{0}(57.02 \mathrm{GHz})$ and $\mathrm{A}^{+} 5_{1} \rightarrow 6_{0}(6.67 \mathrm{GHz})$ transitions have $T \mathrm{~d} V=-10.0 \mathrm{~K} \mathrm{~km} \mathrm{~s}^{-1}$ and $T \mathrm{~d} V=-29.0 \mathrm{~K} \mathrm{~km} \mathrm{~s}^{-1}$, respectively.

Both population inversion and anti-inversion are consistent with the collisional propensities favouring transitions in which $K$ remains unchanged, as recognized by Walmsley et al. (1988) [7] when interpreting their observations of the absorption of the $12.18 \mathrm{GHz}$ transition by the cosmic background radiation field. The lowest $\left(J_{K}\right)$ level of E-type is $1_{-1}$, and of A-type is $0_{0}$, and hence levels with $K=-1$ and $K=0$, in E-type and A-type, respectively, tend to be more highly populated.

\section{Acknowledgements}

The work discussed here derived from an earlier study of the collisional excitation of $\mathrm{NH}_{3}$, on which one of the present authors (DRF) worked closely with Pierre Valiron. Pierre contributed much to our discipline, and he is sorely missed.

\section{References}

1. Codella, C. et al. A\&A, 518, L112 (2010)

\footnotetext{
1 http://massey.dur.ac.uk/drf/methanol
} 
From Quantum Chemistry to Astrophysics: a tribute to Pierre Valiron

2. Rabli, D. \& Flower, D.R. MNRAS, 403, 2033 (2010a)

3. Rabli, D. \& Flower, D.R. MNRAS, 406, 95 (2010b)

4. Rabli, D. \& Flower, D.R. MNRAS, 4112093 (2011)

5. Flower, D.R., Pineau des ForêTs, G., \& Rabli, D. MNRAS, 409, 29 (2010)

6. Voronkov, M.A. et al. MNRAS, 408, 133 (2010)

7. Walmsley, C.M. et al. A\&A, 197, 271 (1988) 